\title{
High Contrast Adaptive Optics for Exoplanet Detections: performance limits and optimal wavefront sensing strategy
}

\section{Olivier Guyon}

Subaru Telescope, National Astronomical Observatory of Japan 659 N. A'ohoku Pl. Hilo, HI 96720, USA

email: guyon@naoj.org

\begin{abstract}
The effects of photon noise, aliasing, wavefront chromaticity and scintillation on the PSF contrast achievable by ground-based adaptive optics (AO) are evaluated for different wavefront sensing schemes. I show that "classical" AO (sensing in the visible, imaging in the near-IR) is limited to about $10^{5} \mathrm{PSF}$ contrast in the central arcsecond because of scintillation chromaticity.

This comparative study shows that a focal-plane based wavefront sensor (WFS), combining wavefront sensing and scientific imaging on the same detector is optimal for high contrast imaging. This approach combines high WFS sensitivity, immunity to aliasing and non common path errors and optical design simplicity. Its theoretical performance is compared to commonly used WFSs, illustrating the advantages of this technique.

I show that such a system can be efficiently used as a second stage after a low-order AO system. Control and data reduction algorithms are presented, as well as possible optical designs incorporating a coronagraph. A laboratory demonstration of this technique is currently being done at Subaru Telescope.
\end{abstract}

Keywords. adaptive optics, speckle, wavefront sensor.

\section{Introduction}

Adaptive optics and coronagraphy are the key enabling technologies for the direct imaging of extrasolar planets. Recent developpements in coronagraphic techniques theoretically allow $\approx 10^{10} \mathrm{PSF}$ contrast level a few $\lambda / d$ from the optical axis. This opens the possibility of direct detection of extrasolar terrestrial planets from space (NASA TPF-C mission).

Atmospheric turbulence, even partially corrected by an AO system, prevents groundbased imaging system from delivering PSFs with such high contrast. In most cases, the achieved PSF contrast limit is therefore set by the AO system performance rather than the coronagraph itself.

In this paper, upper limits on how faint the wings of an Adaptive Optics PSF can be made are computed. In this work, it is assumed that a coronagraph cancels the "static" diffraction (Airy rings). The PSF surface brightness normalized the PSF peak surface brightness is used as a quantifier, and thereafter noted PSF contrast. Actual detection limits for faint companions are not derived in this work, but the PSF contrast is clearly an important factor towards successfull detection:

- Photon noise. Improving the PSF contrast reduces the bright "background" superimposed on a faint companion, and therefore the photon noise that comes with it.

- Speckle noise. Reducing the PSF wings brightness also reduces the speckle noise (Bloemhof et al. 2001, Bloemhof 2003, Aime \& Soummer 2004). 
- Calibration. Detection of a faint companion requires accurate calibration of the PSF wings which needs to be subtracted. This calibration is more difficult if the PSF wings are brighter.

\section{PSF contrast limit}

\subsection{The main terms}

The PSF surface brightness is decomposed in this work in 6 terms, which are not a priory correlated, and can therefore be added together:

- $C_{0}$ : PSF contrast limit imposed by OPD aberrations in uncorrected atmospheric turbulence.

- $C_{1}$ : PSF contrast limit imposed by amplitude aberrations in uncorrected atmospheric turbulence (scintillation).

- $C_{2}$ : PSF contrast limit imposed by residual OPD aberrations after AO correction.

- $C_{3}$ : PSF contrast limit imposed by residual amplitude aberrations after AO correction of OPD and amplitude.

- $C_{4}$ : PSF contrast limit imposed by the differential OPD between the WFS and imaging wavelengths. This term is caused by the chromaticity of Fresnel propagation.

- $C_{5}$ : PSF contrast limit imposed by the differential scintillation between the WFS and imaging wavelengths. This term is caused by the chromaticity of Fresnel propagation.

- $C_{6}$ : PSF contrast limit imposed by the differential OPD between the WFS and imaging wavelengths. This term is caused by the chromaticity of the refraction index of air.

With an AO system correcting the wavefront phase (but without scintillation correction), the PSF surface brightness is $C=C_{1}+C_{2}+C_{4}+C_{6}$. If phase and amplitude are corrected, the PSF surface brightness becomes $C=C_{2}+C_{3}+C_{4}+C_{5}+C_{6}$.

Analytical expressions for each of these terms are derived in Guyon (2005) for observations at the zenith, and results shown in this paper are derived from these expressions. The relative importance of these terms is shown in figure 1 in a particular example (which details can be found in Guyon (2005)).

It should be noted that terms $C_{2}$ and $C_{3}$ are function of the wavefront sensor efficiency, which is defined and computed for several WFSs in Guyon (2005). This topic will be discussed in more detail in $\S 3$.

\subsection{Wavelength choices}

In a well-optimized AO system, able to correct both the phase and amplitude of the incoming wavefront, the residual PSF surface brightness due to chromatic effects $\left(C_{4}\right.$, $C_{5}$ and $\left.C_{6}\right)$ dominates the contribution due to photon noise and time lag $\left(C_{2}\right.$ and $\left.C_{3}\right)$ if $\lambda_{W F S} \neq \lambda_{P S F}$. In the example given in figure 1 , where $\lambda_{W F S}=0.55 \mu m$ and $\lambda_{P S F}=$ $1.6 \mu \mathrm{m}$, this difference exceeds 3 orders of magnitudes within the central arcsecond.

Unless the chromatic effects can be calibrated (which appears to be feasible for $C_{6}-$ Fusco T., private communication), high PSF contrast within the central arcsecond therefore requires $\lambda_{W F S} \approx \lambda_{P S F}$. It is especially interesting to notice that in the example given in figure 1 , the contribution of scintillation to the PSF surface brightness is quite close to the contribution due to scintillation chromaticity.

\section{Wavefront sensor choice}

The results shown in figure 1 assume a wavefront sensor (WFS) with optimal efficency, which makes optimal use of every detected photon to reconstruct the incoming 


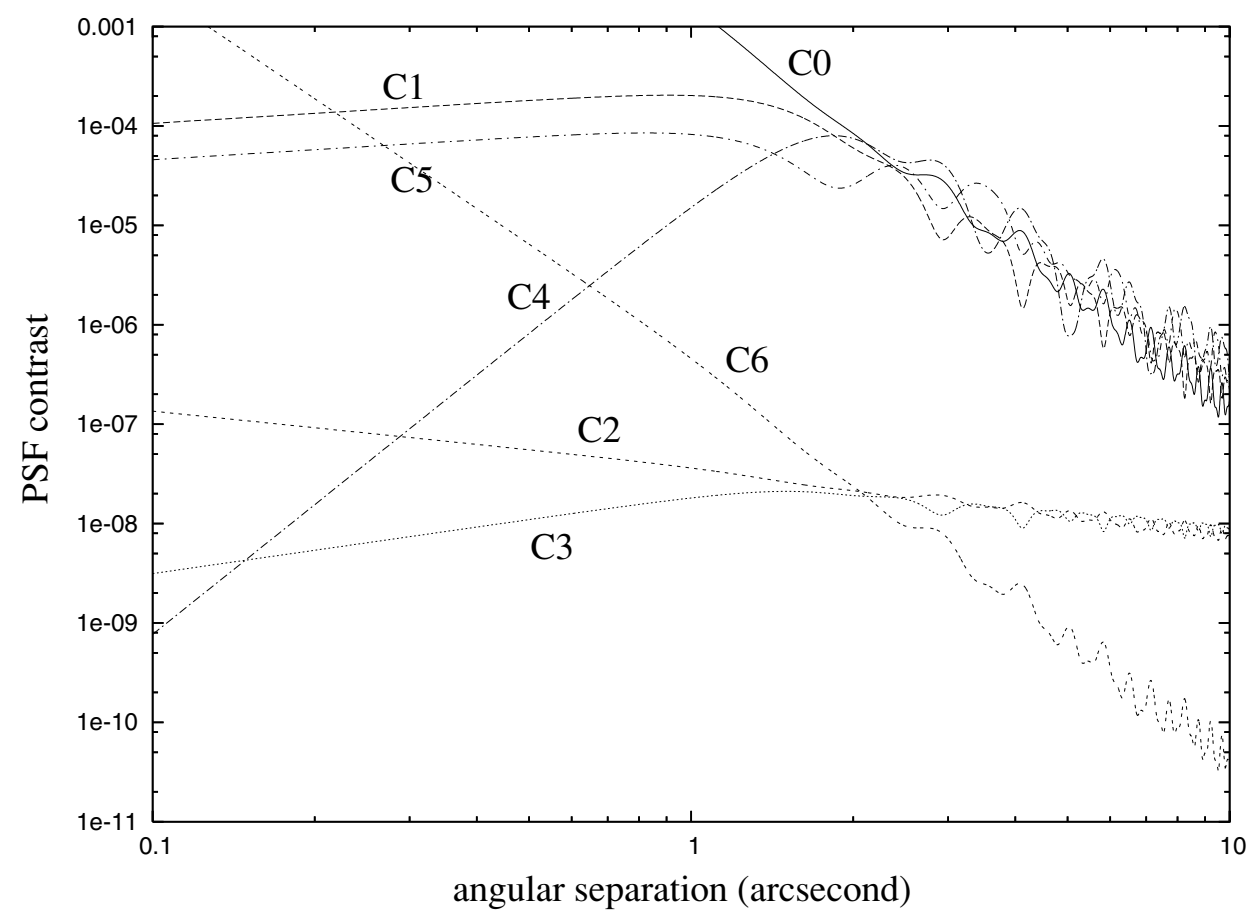

Figure 1. Main sources of PSF contrast limit as a function of angular separation for an $8 \mathrm{~m}$ telescope. C0 shows what would be the PSF contrast if the atmospheric turbulence were uncorrected. $\mathrm{C} 1$ is the atmospheric scintillation. The residual phase (C2) and amplitude (C3) errors after correction if an "ideal" WFS are shown here for a mv=5 guide star. Chromatic effects C4/C5 (chromaticity of OPD and amplitude due to diffractive propagation through the atmosphere) and C6 (chromaticity of the air refraction index) are shown for $\lambda_{W F S}=0.55 \mu m$ and $\lambda_{P S F}=1.6 \mu \mathrm{m}$. Exact details of this simulation and analytical expressions for these terms can be found in Guyon (2005).

wavefront's phase and amplitude. As detailed in Guyon (2005), the efficiency of a WFS can be quantified by the parameters $\beta_{p}$ and $\beta_{a}$ for respectively pupil phase and pupil amplitude measurements. In most WFSs, $\beta_{p}$ and $\beta_{a}$ are a function of the spatial frequency in the pupil plane, or, equvalently, the angular distance to the PSF center.

As can be seen in figure 2, WFSs fall into 2 categories:

- WFSs sensitive to "noise propagation": Shack-Hartmann (SHWFS), Curvature (CWFS) and Modulated Pyramid (MPYRWFS). In these WFSs, the efficiency is poor at small angular separation, especially if the number of sensing elements is high.

- WFSs insensitive to "noise propagation": Pupil Plane Mach Zehnder (PPMZWFS), Focal Plane (FPWFS, a type of phase diversity measurement), Zernike (ZWFS) and Fixed Pyramid (FPYRWFS). These WFSs maintain a very good efficency at all spatial frequencies simultaneously.

WFSs insensitive to noise propagation appear theoretically superior for high contrast imaging, but offer limited dynamical range, poor linearity, and in some cases limited spectral bandwidth due to chromatic effects. They are therefore adapted to the highStrehl regime where linearity can be maintained (residual WF errors less than 1 radian at the WFS wavelength).

Although WFSs which are sensitive to noise propagation offer poor efficency, they are usually very linear and are therefore more robust if the residual WF errors are large (which is usually the case in current AO systems). It should be noted that with the proper 


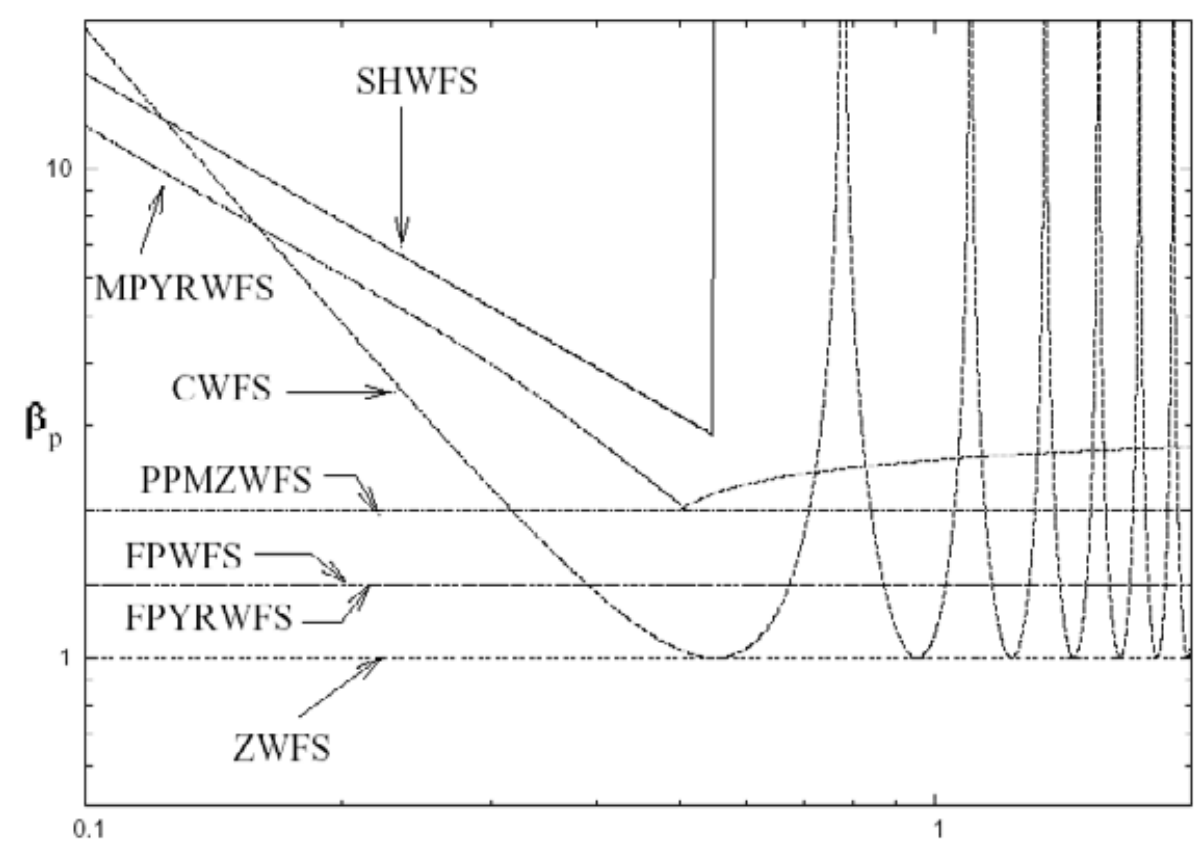

Figure 2. Efficiency of different WFSs for the measurement of phase aberrations in the pupil plane. The efficiency $\beta_{p}$ is optimal when equal to 1 . The number of photons required to reach a given measurement accuracy is proportionnal to $\beta_{p}{ }^{2}$. The efficiency is shown as a function of angular separation for a $8 \mathrm{~m}$ telescope (with $\lambda_{W F S}=0.55 \mu \mathrm{m}$ and $\lambda_{P S F}=1.6 \mu \mathrm{m}$ ). Exact details of this simulation and analytical expressions for these terms can be found in Guyon (2005).

choice of actuator sampling in the pupil plane, and by avoiding the use of a coronagraph which is too sensitive to low odrer aberrations, such WFSs can still successfully be used to efficiently "clean" the PSF at a given angular distance. This is illustrated in figure 2 where these WFSs were tuned to deliver optimal efficiency at a 0.5 " angular separation.

\section{Conclusion}

\subsection{Wavefront sensing \& control strategies, coronagraphy}

To approach the PSF contrast limit theoretically given by photon noise (terms $C_{2}$ and $C_{3}$ for $\beta=1$ ), a high performance WFS should be used at the imaging wavelength. A phase diversity technique (referred to Focal Plane Wavefront Sensor, or FPWFS, in this work), using the same focal plane detector array for wavefront sensing and scientific imaging, seems ideal for this application. Its main advantages are (1) excellent efficiency at all spatial frequencies (2) low aliasing (3) absence of non-common path errors. The DM, upstream of the coronagraph, introduces the diversity necessary for wavefront reconstruction. The wavefront should preferably be "cleaned" by a first stage AO system prior to the coronagraph/ FPWFS unit. This scheme is shown in figure 3 .

Ideally, the second stage AO system needs to be fast (bandwidth $>10 \mathrm{kHz}$ on bright stars), requiring a frame rate of about $100 \mathrm{kHz}$ : as soon as a few photons per focal plane speckle are detected, a command should be sent to the fast DM (Angel 2003). A fast low readout noise detector is therefore essential for this application. An equally challenging aspect of this system is the required computation speed for the second stage AO system. 


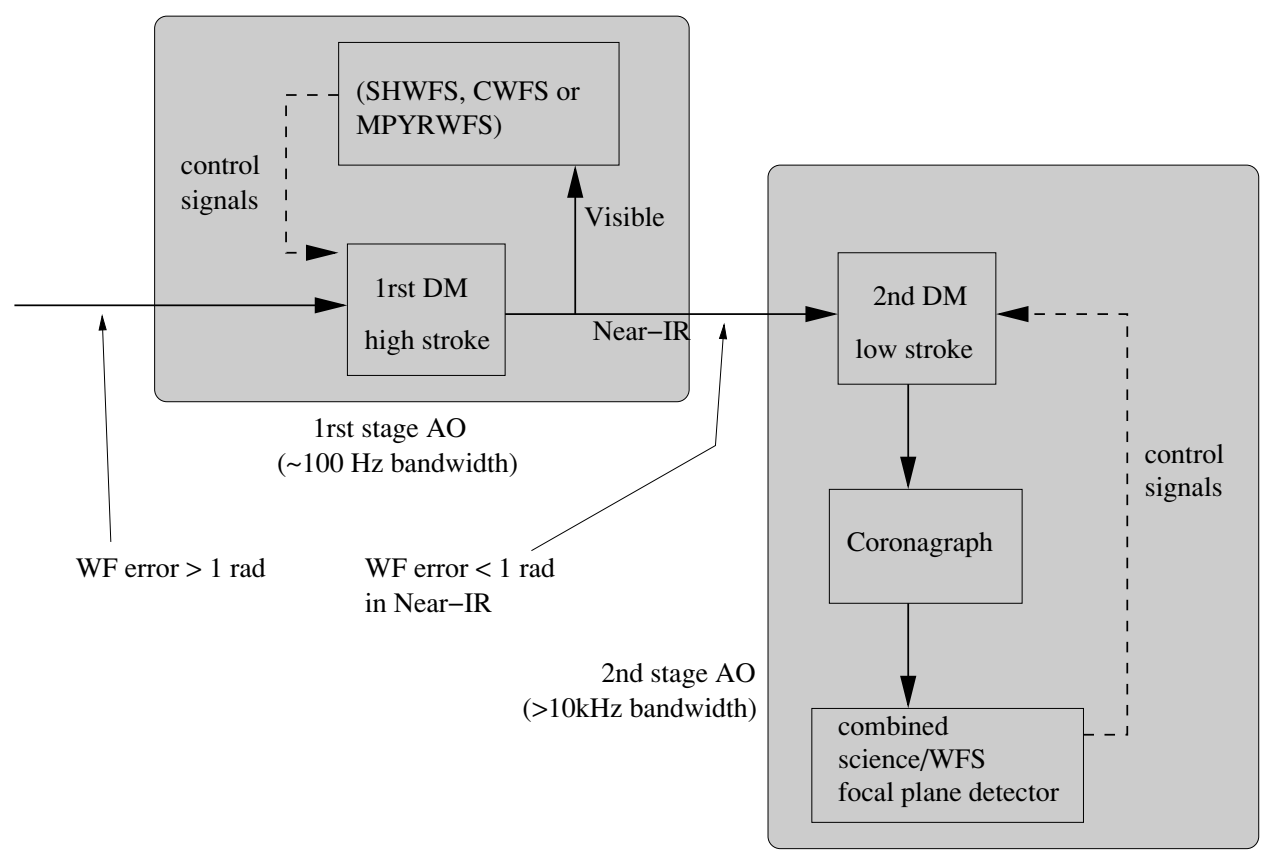

Figure 3. Schematic representation of a 2 stages AO system + coronagraph optimized for high contrast imaging. The first stage AO system can be a conventionnal Shack-Hartmann or Curvature based system, while the second faster AO system is a focal plane WFS. In this example, the science wavelength is "Near-IR", but the same scheme could be used for visible imaging (in which case light might have to be share with the first stage AO system WFS).

\subsection{Imaging extrasolar terrestrial planets from the ground?}

An Earth-sized planet is about $10^{-10}$ the brightness of its central star, and a Jupiterlike planet is only about 10 times brighter. The angular separation for these targets is less than 1". Even the optimistic PSF contrast limit derived in this work are quite far from this level. One way to improve the PSF contrast is to increase the telescope diameter: this concentrates the planet's light in a tighter core $(\operatorname{size} \lambda / D)$ which better stands out of the PSF wings. As shown in figure 4, under favorable conditions, a $10^{8}$ contrast is theoretically achievable at 0.2 " with a $60 \mathrm{~m}$ diameter telescope and a high quality coronagraph which suppress the Airy pattern by 3 orders of magnitude. The residual speckles then need to be calibrated to a $10 \%$ accuracy to recover a Jupiter-like planet: this calibration requirement is not particularly challenging, especially if most of the speckles average into a smooth background in long exposures.

With the same assumptions, direct detection of an Earth-like planet would require 1\% speckle calibration accuracy, which, although challenging, is quite concievable. In this case, the required number of planetary photon for a $\mathrm{SNR}=7$ detection is 5000: with a $60 \mathrm{~m}$ diameter telescope, the detection would not require prohibitive exposure times.

\section{References}

Angel, J.R.P. 2003, ASP Conf. Ser, 294, 543-556, "Scientific Frontiers in Research on Extrasolar Planets", eds. S. Seager and D. Deming, Washington D.C. 2003

Bloemhof, E.E., Dekany, R.G., Troy, M., \& Oppenheimer, B.R. 2001, ApJ, 558, L71

Bloemhof, E.E. 2003 ApJ, 582, L59

Guyon, O. 2005, ApJ 629, 592 


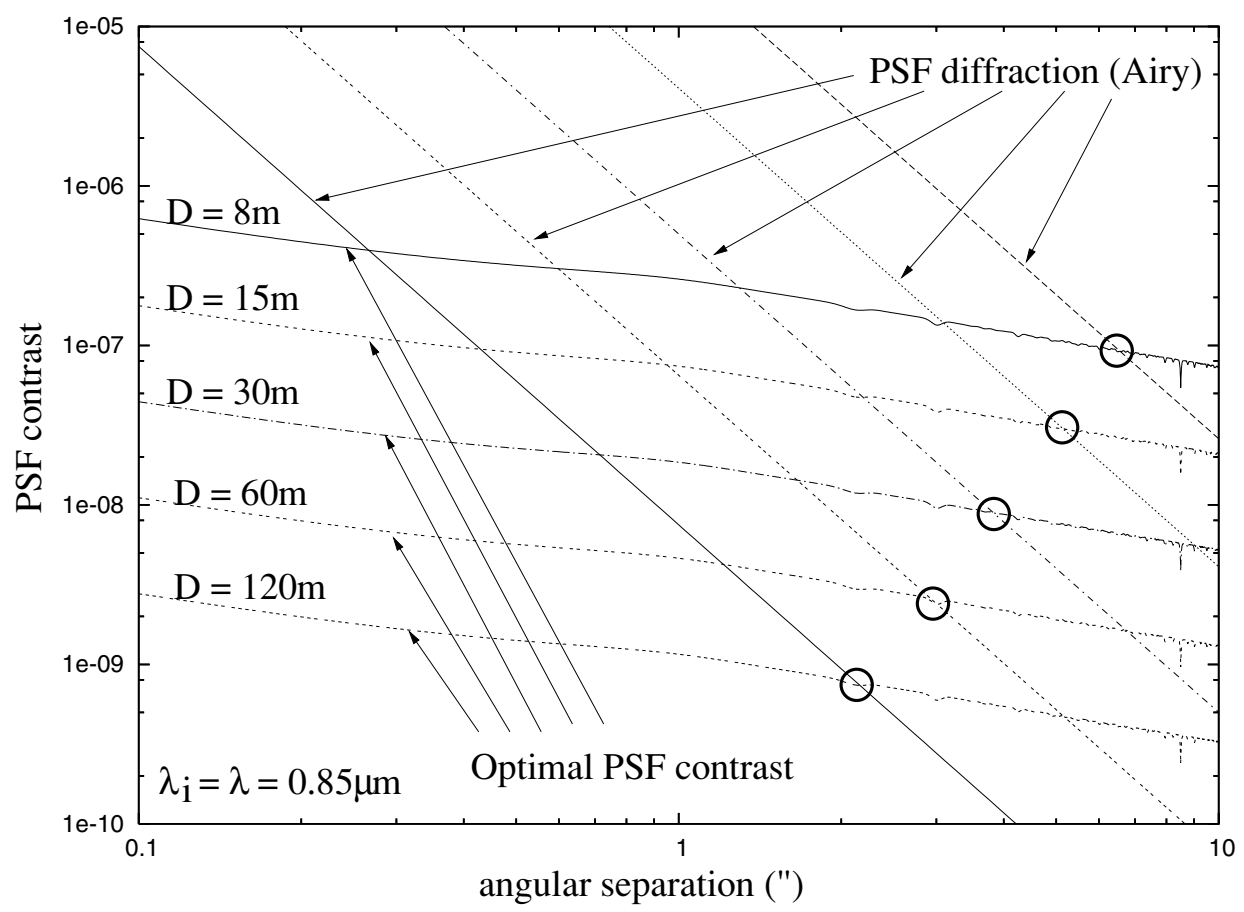

Figure 4. PSF contrast with an optimal WFS $(\beta=1)$ and Airy pattern diffraction for $8 \mathrm{~m}, 15 \mathrm{~m}$, $30 \mathrm{~m}, 60 \mathrm{~m}$ and $120 \mathrm{~m}$ diameter telescopes. The black circles mark the points where the theoretical PSF contrast achievable with a perfect coronagraph equals the Airy pattern diffraction contrast level.

\section{Discussion}

FuscO: Another important effect not mentionned in your work is the chromatic shear of the wavefront due to atmospheric dispersion. This effect can easily dominate others when $\lambda_{W F S} \neq \lambda_{P S F}$ and observations are not made at Zenith.

Fusco: It should be possible to correct for the term $C_{6}$ (chromaticity of the refraction index of air) since it is a well known scaling constant. 